\title{
Nonthermal Quantum Channels as a Thermodynamical Resource
}

\author{
Miguel Navascués ${ }^{1}$ and Luis Pedro García-Pintos ${ }^{2}$ \\ ${ }^{1}$ Department of Physics, Bilkent University, Ankara 06800, Turkey \\ ${ }^{2}$ School of Mathematics, University of Bristol, University Walk, Bristol BS8 ITW, United Kingdom
}

(Received 19 January 2015; revised manuscript received 31 March 2015; published 2 July 2015)

\begin{abstract}
Quantum thermodynamics can be understood as a resource theory, whereby thermal states are free and the only allowed operations are unitary transformations commuting with the total Hamiltonian of the system. Previous literature on the subject has just focused on transformations between different state resources, overlooking the fact that quantum operations which do not commute with the total energy also constitute a potentially valuable resource. In this Letter, given a number of nonthermal quantum channels, we study the problem of how to integrate them in a thermal engine so as to distill a maximum amount of work. We find that, in the limit of asymptotically many uses of each channel, the distillable work is an additive function of the considered channels, computable for both finite dimensional quantum operations and bosonic channels. We apply our results to bound the amount of distillable work due to the natural nonthermal processes postulated in the Ghirardi-Rimini-Weber (GRW) collapse model. We find that, although GRW theory predicts the possibility of extracting work from the vacuum at no cost, the power which a collapse engine could, in principle, generate is extremely low.
\end{abstract}

PACS numbers: 05.30.-d, 03.65.Ta, 03.67.-a, 05.70.-a

The field of quantum thermodynamics has seen a surge in interest in the past years, with increasing attention towards testing the validity of the rules of classical thermodynamics in the quantum regime. A major topic within thermodynamics is that of extracting work out of a given system and the optimal way to perform this. One way this has been approached in the quantum case was by considering it from the perspective of a resource theory.

The idea of a resource theory of thermodynamics is to assume one has unlimited access to thermal baths (i.e., Gibbs states of a fixed temperature $T$ ), the freedom to apply any energy conserving unitary on system plus the bath (any unitary $V$ that commutes with the total Hamiltonian), and the possibility of discarding part of the system or bath (i.e., apply partial traces). These rules are imported from classical thermodynamics, where one assumes access to infinite baths of constant temperature and any evolution where energy is conserved.

As a matter of fact, resource theories have been very useful in different topics within quantum information theory [1-4]. The idea is similar to above: considering free access to certain operations and/or states, any state and/ or operation that is not in the above set can, in principle, be used as a resource.

This Letter complements previous research in quantum thermodynamics by accommodating the possibility of considering nonthermal maps, or channels, as a resource.

Physical operations are represented by quantum channels, i.e., completely positive trace preserving maps $\Omega: B(\mathcal{H}) \rightarrow$ $B\left(\mathcal{H}^{\prime}\right)$ acting on a state space $B(\mathcal{H})$. For simplicity, we will assume that the input and output spaces (and, as we will see later, Hamiltonians) of each channel are the same, although the results can be easily generalized.

Unitary evolution is a particular instance of a quantum channel, determined by the evolution operator. However, quantum channels allow us to express more general evolutions. For instance, any unitary interaction of a system with an ancilla (or environment) generates a quantum channel, given by

$$
\Omega(\rho)=\operatorname{Tr}_{A}\left[V \rho \otimes \sigma_{A} V^{\dagger}\right]
$$

where $\sigma_{A}$ is the state of the ancilla, and $V$ is some unitary operator In fact, it can be shown that any channel can be generated via the above procedure [5].

If, in the above expression, the ancilla is in a Gibbs state of temperature $T$ and the unitary $V$ commutes with the total Hamiltonian $H_{T}=H_{S} \otimes \mathbb{1}_{A}+\mathbb{1}_{S} \otimes H_{A}$ of the targetancilla system, the resulting map is called a thermal channel. These will be the free operations in our theory, while any nonthermal map $\Omega$ will be considered as a resource.

In this scenario, we define quantum work $W$ as the process of exciting a two-level system with Hamiltonian $H=W|1\rangle\langle 1|$ from its ground state $|0\rangle$ to the excited state $|1\rangle$ [6]. Different authors have explored how much work one can extract from a nonthermal quantum state [6-12]. When we regard the maximum average work as a figure of merit, a quantum generalization of the classical free energy naturally emerges $[6,7,12]$

$$
F(\rho)=U(\rho)-K_{B} T S(\rho) .
$$

Here, $\rho$ is the state of the system from which we wish to extract work; $U(\rho)=\operatorname{Tr}[\rho H]$, its average energy; and 
$S(\rho)=-\operatorname{Tr}[\rho \log \rho]$, its von Neumann entropy. $K_{B}$ is Boltzmann's constant. The maximum amount of work one can extract (on average) from the state $\rho$ can then be shown to be $F(\rho)-F\left(\tau_{\text {th }}\right)$, where $\tau_{\text {th }}$ represents the thermal state at temperature $T$.

In this Letter, we want to address the following related problem: suppose we want to build a thermal engine, where we are allowed to integrate a number of nonthermal gates $\left\{\Omega_{i}\right\}_{i=1}^{N}$, each of which is assumed to act on a system with Hamiltonian $H_{i}$. More specifically, our machine can make free use of any amount of thermal states and operations, and we can invoke one use of each of the channels $\left\{\Omega_{i}\right\}_{i=1}^{N}$, in any order we want at any step. We are also allowed to use catalysts; i.e., we can use any number of nonthermal states, as long as we return them in the end. Under these conditions, what is the maximum amount of work that our device can extract?

There are two ways to approach this problem: (1) We can restrict to thermodynamical processes which distill work deterministically, i.e., always the same amount. The corresponding deterministic extractable work can then be shown to behave very badly: not only is it not additive, but it can be superactivated. That is, there exist channels $\Omega$ such that no work can be distilled from a single use, but two uses of the channel can be combined to produce a nonzero amount of deterministic work (see the Supplemental Material [13]). (2) Alternatively, we can consider thermodynamical processes which generate a given amount of work with high probability. Here, the figure of merit would be the maximum amount of work that can be distilled asymptotically (on average) when we have access to $n$ uses of each channel.

We will follow the second approach: In the next pages, we will show that the asymptotically extractable work is upper bounded by $\sum_{i=1}^{N} W\left(\Omega_{i}, H_{i}\right)$, where

$$
W(\Omega, H) \equiv \max _{\rho} \Delta F(\rho, \Omega),
$$

with $\Delta F(\rho, \Omega)$ denoting the free energy difference between the states $\Omega(\rho)$ and $\rho$, i.e.,

$$
\Delta F(\rho, \Omega) \equiv \operatorname{Tr}[\{\Omega(\rho)-\rho\} H]-K_{B} T[S\{\Omega(\rho)\}-S(\rho)] .
$$

The quantity $W(\Omega, H)$ will be called the distillable work of channel $\Omega$. From the inequality $\Delta F\left(\tau_{\text {th }}, \Omega\right) \geq 0$, it follows that $W(\Omega, H) \geq 0$ for any $\Omega$.

The bound $\sum_{i=1}^{N} W\left(\Omega_{i}, H_{i}\right)$ can be achieved asymptotically via a simple protocol where we prepare suitable initial states $\sigma_{\text {cat }}$ (the catalysts) maximizing Eq. (3) for each channel, and then, let each channel act over its corresponding maximizer. The result of this protocol will be a state with free energy $F\left(\sigma_{\text {cat }}\right)+\sum_{i=1}^{N} W\left(\Omega_{i}, H_{i}\right)$. Given access to $n$ uses of each channel, we can, thus, prepare $n$ copies of the latter state, whose free energy can be converted to work via thermal operations using the protocol depicted in [7]. Following [7], part of this Letter [roughly $n F\left(\sigma_{\text {cat }}\right)$ ] can then be used to regenerate the catalysts up to a small error [14]. The average work extracted with this procedure (namely, the total work divided by $n)$ is, thus, given by $\sum_{i=1}^{N} W\left(\Omega_{i}, H_{i}\right)$.

Note, though, that, unless the catalysts are diagonal in the energy basis, an extra amount of coherence, sublinear in $n$, may be needed to rebuild them (see Appendix E of [7]). More specifically, for each energy transition $E_{s} \rightarrow E_{t}$ in the Hamiltonian $H_{i}$, the protocol proposed in [7] requires a system with Hamiltonian $H_{i}^{s, t}=\sum_{k=0}^{O(m)}\left(E_{s}-E_{t}\right) k|k\rangle\langle k|$ in state $(1 / \sqrt{m}) \sum_{k=0}^{m}|k\rangle$, with $m$ sublinear in the number $n$ of uses of each channel. Like the catalyst states, at the end of the protocol, such "coherent states" will be approximately rebuilt with vanishing error.

In order to prove the above result, and some later ones, the next lemma will be invoked extensively.

Lemma 1.-Let $\sigma^{(N)}$ be an $N$-partite quantum state, and let $\left\{\Omega_{i}\right\}_{i=1}^{N}$ be a collection of $N$ single-site quantum channels. Defining $\Omega_{1, \ldots, N} \equiv \bigotimes_{i=1}^{N} \Omega_{i}$, we have

$$
\sum_{i=1}^{N} S\left(\sigma_{i}\right)-S\left[\Omega_{i}\left(\sigma_{i}\right)\right] \geq S\left(\sigma^{(N)}\right)-S\left[\Omega_{1, \ldots, N}\left(\sigma^{(N)}\right)\right] .
$$

The proof is a straightforward application of the contractivity of the relative entropy [15].

An almost immediate consequence of Lemma 1 is that $W(\Omega, H)$, as defined by Eq. (3), has the remarkable property of being additive. That is, if the bipartite system 12 is described by the Hamiltonian $H_{12}=H_{1} \otimes \mathbb{1}_{2}+\mathbb{1}_{1} \otimes H_{2}$, and the channels $\Omega_{1}$ and $\Omega_{2}$ act on the respective Hilbert spaces $\mathcal{H}_{1}, \mathcal{H}_{2}$, then, $W\left(\Omega_{1} \otimes \Omega_{2}, H_{12}\right)=W\left(\Omega_{1}, H_{1}\right)+$ $W\left(\Omega_{2}, H_{2}\right)$.

Indeed, let $\Omega_{12} \equiv \Omega_{1} \otimes \Omega_{2}$ act on the bipartite state $\rho_{12}$. By choosing $\rho_{12}=\rho_{1} \otimes \rho_{2}$ in (3), we trivially have $W\left(\Omega_{1} \otimes \Omega_{2}, H_{12}\right) \geq W\left(\Omega_{1}, H_{1}\right)+W\left(\Omega_{2}, H_{2}\right)$, since maximizing over states in 12 is more general than maximizing over 1 and 2 independently. Let us then focus on the opposite inequality. By Lemma 1, we have

$$
\sum_{i=1,2} S\left(\rho_{i}\right)-S\left[\Omega_{i}\left(\rho_{i}\right)\right] \geq S\left(\rho_{12}\right)-S\left[\Omega_{12}\left(\rho_{12}\right)\right] .
$$

Substituting into (3) gives

$$
\sum_{i=1,2} \Delta F\left(\rho_{i}, \Omega_{i}\right) \geq \Delta F\left(\rho_{12}, \Omega_{12}\right) \quad \forall \rho_{12} .
$$

It follows that $\sum_{i=1,2} W\left(\Omega_{i}, H_{i}\right) \geq W\left(\Omega_{12}, H_{12}\right)$.

We are now ready to prove that $W(\Omega, H)$ quantifies the maximum (average) amount of work one can extract from channel $\Omega$.

Proposition 1.-Let $\left\{\Omega_{i}\right\}_{i=1}^{N}$ be a set of quantum channels, defined over different quantum systems with 
Hamiltonians $\left\{H_{i}\right\}_{i=1}^{N}$. Suppose we integrate $n$ uses of all such channels in a thermal engine $\mathcal{T}_{n}$ that produces a net amount of work $W_{n}$ with probability $1-\epsilon_{n}$. Let us, further, assume that the probability of failure vanishes in the limit of large $n$, i.e., $\lim _{n \rightarrow \infty} \epsilon_{n}=0$. Under these conditions, the average asymptotic work $\bar{W} \equiv \limsup _{n \rightarrow \infty}\left(W_{n} / n\right)$ satisfies

$$
\bar{W} \leq \sum_{i=1}^{N} W\left(\Omega_{i}, H_{i}\right) .
$$

As indicated above, this bound is achievable with the use of catalysts and a sublinear amount of quantum coherence.

Proof.-In any protocol for work extraction, the initial state of the system will be given by the catalysts $\sigma_{\text {cat }}$, a number of thermal states $\tau_{\text {th }}$, and the work system in state $|0\rangle_{w}$. The initial state of the system is, hence, $\rho_{0} \equiv \sigma_{\text {cat }} \otimes \tau_{\text {th }} \otimes|0\rangle\left\langle\left. 0\right|_{w}\right.$, with free energy $F\left(\sigma_{\text {cat }}\right)+F\left(\tau_{\text {th }}\right)$.

Suppose that now we apply a sequence of energyconserving unitaries. At time $t$, the state of the overall system is $\rho_{t}$, and we apply the channel $\Omega_{s(t)}$ over part of the whole system, possibly followed by some other thermal operation. Let us analyze how the free energy of $\rho_{t}$ can increase in the above step. Calling $H_{T}$ the Hamiltonian of the whole system, from the definition of $W(\Omega, H)$ and the additivity of the distillable work we have

$$
\Delta F\left(\Omega_{s(t)} \otimes \mathbb{1}, \rho_{t}\right) \leq W\left(\Omega_{s(t)} \otimes \mathbb{1}, H_{T}\right)=W\left(\Omega_{s(t)}, H_{s(t)}\right) .
$$

Now, any intermediate energy-conserving unitary in between the use of any two of the channels $\left\{\Omega_{i}\right\}_{i=1}^{N}$ will keep the free energy of the overall system constant. Calling $\bar{\rho}$ the state of the system at the end of the protocol, hence, we have

$$
F(\bar{\rho}) \leq n \sum_{i=1}^{N} W\left(\Omega_{i}, H_{i}\right)+F\left(\sigma_{\text {cat }}\right)+F\left(\tau_{\text {th }}\right) .
$$

From the subadditivity of the von Neumann entropy, it follows that $F(\bar{\rho}) \geq F\left(\bar{\rho}_{\text {cat }}\right)+F\left(\bar{\rho}_{\text {th }}\right)+F\left(\bar{\rho}_{w}\right)$, where $\bar{\sigma}_{\text {cat }}, \bar{\rho}_{\text {th }}, \bar{\rho}_{w}$ are, respectively, the reduced density matrices of the catalyst, thermal, and work systems.

At the end of the protocol, the catalyst must be regenerated, i.e., $\bar{\sigma}_{\text {cat }}=\sigma_{\text {cat }}$. Also, $F\left(\bar{\rho}_{\text {th }}\right) \geq F\left(\tau_{\text {th }}\right)$. It follows that the free energy of the work system is bounded by $n \sum_{i=1}^{N} W\left(\Omega_{i}, H_{i}\right)$.

This system is expected to end up in state $|1\rangle$ with probability $1-\epsilon_{n}$, i.e., $\bar{\rho}_{w}=\left(1-\epsilon_{n}\right)|1\rangle\langle 1|+\epsilon_{n} \bar{\sigma}$. It follows that $F\left(\bar{\rho}_{w}\right) \geq\left(1-\epsilon_{n}\right) W_{n}-K_{B} T h\left(\epsilon_{n}\right)$, with $h(p)=$ $-p \ln (p)-(1-p) \ln (1-p)$. In the asymptotic limit, with $n \rightarrow \infty, \epsilon_{n} \rightarrow 0$, the average asymptotic work $\limsup \left(W_{n} / n\right)$ is, hence, bounded by $\sum_{i=1}^{N} W\left(\Omega_{i}, H_{i}\right)$.

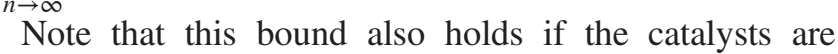
recovered up to an error, as long as $F\left(\sigma_{\text {cat }}\right)-F\left(\bar{\sigma}_{\text {cat }}\right) \leq o(n)$.
This result allows us to quantify the work extraction capabilities of different channels. One can check, for instance, that no work can be distilled from a dephasing channel. Meanwhile, for a two-level system with Hamiltonian $H=E|1\rangle\langle 1|, E>0$, the channel that takes any state to the excited state $|1\rangle$ provides the highest distillable work.

Gaussian channels.-If our target system is infinite dimensional, in principle, there may exist quantum states possessing an infinite amount of energy. If we regard such states as unphysical, we should replace the maximization in Eq. (3) by an optimization over all states of finite energy. Hence, the resulting quantity will bound the maximum amount of work generated in physically conceivable quantum engines, where the overall state of the system always has a finite amount of energy.

In infinite dimensional systems, Gaussian quantum channels have a special relevance: they are easy to implement in the lab, and are extensively used to model particle interactions with a macroscopic environment. They are defined as channels which, when composed with the identity map, transform Gaussian states into Gaussian states, the latter being those states with a Gaussian Wigner function [16]. An $m$-mode Gaussian state is completely defined via its displacement vector $d_{i}=\left\langle R_{i}\right\rangle$ and covariance matrix $\gamma_{i j}=\left\langle\left\{R_{i}-d_{i} \mathbb{1}, R_{j}-d_{j} \mathbb{\square}\right\}_{+}\right\rangle$, where $\left(R_{1}, R_{2}, \ldots, R_{2 m}\right) \equiv\left(Q_{1}, P_{1}, \ldots, Q_{m}, P_{m}\right)$ are the optical quadratures. The action of a Gaussian channel is fully specified by its action over the displacement vector and covariance matrix, given by

$$
d \rightarrow X d+z, \quad \gamma \rightarrow X \gamma X^{T}+Y,
$$

where $Y+i \sigma-i X^{T} \sigma X \geq 0$. Here, $\sigma$ denotes the symplectic form $\sigma=\oplus_{i=1}^{m}\left(\begin{array}{cc}0 & 1 \\ -1 & 0\end{array}\right)$. If the Hamiltonian of the system under study happens to be a quadratic function of the optical quadratures, i.e., $H=\vec{R}^{T} G \vec{R}+\vec{h} \cdot \vec{R}$, for some real symmetric matrix $G$ and real vector $\vec{h}$, then the average energy of a state with displacement vector $\vec{d}$ and covariance matrix $\gamma$ is given by $E=\frac{1}{2} \operatorname{Tr}[G \gamma]+\vec{d}^{T} G \vec{d}+\vec{h} \cdot \vec{d}$. Hence, states with finite energy correspond to states with finite first and second moments.

When the quadratic Hamiltonian has no zero energy modes (that is, when $G>0$ ), Proposition 1 allows us to easily classify generic Gaussian channels according to their capacity to generate an infinite amount of work. Indeed, for $X^{T} G X-G \not \leq 0$, the channel's distillable work is unbounded: this can be seen by inputting a sequence of Gaussian states with a constant covariance matrix but increasing displacement vector parallel to any positive eigenvector of $X^{T} G X-G$. Conversely, as we show in the Supplemental Material [13], for channels satisfying $X^{T} G X-G<0$, only a finite amount of work can be distilled. 
For such channels, there is still the dilemma of how much work can be extracted. The next Proposition greatly simplifies this problem by showing that, for Gaussian channels $\Omega$, the maximization in (3) can be restricted to Gaussian states.

Proposition 2.-Consider a continuous variable quantum system of $m$ modes, with quadratic Hamiltonian $H$, let $\Omega$ be a Gaussian channel mapping $m$ modes to $m$ modes, and denote by $\mathcal{G}$ the set of all $m$-mode Gaussian states. Then,

$$
W(\Omega, H)=\max _{\rho \in \mathcal{G}} \Delta F(\rho, \Omega) .
$$

The proposition can be proven by combining Lemma 1 with the "gaussification" protocol described in [17], see the Supplemental Material [13].

Since $W(\Omega, H)$ just involves an optimization over a finite set of parameters subject to positive semidefinite constraints, (in principle) it can be computed exactly for any Gaussian channel $\Omega$.

One application: collapse engines.-In order to address the measurement problem [18], and, independently, the decoherence effects that a quantum theory of gravity could impose on the wave function $[19,20]$, different authors have proposed that closed quantum systems should evolve according to the Lindblad equation

$$
\frac{d}{d t} \rho_{t}=-\frac{i}{\hbar}\left[H, \rho_{t}\right]-\frac{\Lambda}{4}\left[X,\left[X, \rho_{t}\right]\right],
$$

where $X$ is the position operator for the particle considered.

The effect of the nonunitary term is a suppression of coherences in the position basis, effectively destroying quantum superpositions. The value of the constant $\Lambda$, which can be interpreted as the rate at which this localization process occurs, depends on the particular theory invoked to justify Eq. (13). In the Ghirardi-Rhimini-Weber (GRW) theory [18], the localization process is postulated to solve the measurement problem in quantum mechanics. To achieve this goal and avoid contradictions with past experimental results, $\Lambda$ must be roughly between $10^{-2} \mathrm{~s}^{-1} \mathrm{~m}^{-2}$ and $10^{6} \mathrm{~s}^{-1} \mathrm{~m}^{-2}$, according to latest estimations [21].

Note that the above evolution is nonthermal. Hence, it could be used in principle to extract work from nothingness by means of a suitable thermal engine. We will call such a hypothetical device a collapse engine.

To connect this to our previous setting, notice that the evolution equation (13) defines a quantum channel

$$
\Omega_{\delta t}\left(\rho_{t}\right)=\rho_{t+\delta t},
$$

with $\rho_{t}$ the solution of Eq. (13) for the initial state $\rho_{0}$.

We suppose that the particle under consideration is subject to a harmonic potential, i.e., $H=\left(m \omega^{2} / 2\right) X^{2}+$ $(1 / 2 m) P^{2}$, and that, despite the GRW dynamics, the bath's temperature $T$ is constant. A physical justification for this last assumption is that the temperature-increasing GRW dynamics is countered by radiation from the bath into outer space. Hence, as a function of time, the temperature will converge to a stationary value $T=T_{\text {eq }}$ above the temperature of the cosmic microwave background (CMB) [22]. Finally, we suppose that our set of resource operations remains the same: that is, in spite of the modified Schrödinger equation (13), we can still switch on and off any unitary interaction that commutes with the total energy of the system. Notice that the GRW dynamics can be modeled by an open system approach, where the particle is interacting with some unknown system such that the resulting evolution is given by (13). From this viewpoint, we are simply assuming that we still have the capacity to interact with the system in the usual way.

In these conditions, we wish to find the maximum amount of work that a collapse engine could extract if it had access to the evolution equation (13) for a finite amount of time $t$. From Proposition 1, this amounts to computing $\lim _{\delta t \rightarrow 0}(t / \delta t) W\left(\Omega_{\delta t}, H\right)$.

First, notice that we can (reversibly) evolve the system with the Hamiltonians $H$ or $-H$, since this corresponds to a thermal operation. This implies that we can ignore the first term in the right hand side of (13), and what remains is a Gaussian channel given by

$$
d \rightarrow d, \quad \gamma \rightarrow \gamma+\left(\begin{array}{cc}
0 & 0 \\
0 & \frac{\hbar^{2} \Lambda \delta t}{2}
\end{array}\right) .
$$

It follows that the energy of any input state will increase by $\Delta U \equiv\left(\hbar^{2} \Lambda / 4 m\right) \delta t$. From Proposition 2, we can estimate the entropy increase by just considering Gaussian states. Now, the entropy of a one-mode Gaussian state is an increasing function of the determinant of its covariance matrix [16], which, by the above equation, can only increase with time. Hence, $W\left(\Omega_{\delta t}, H\right) \leq \Delta U$.

On the other hand, suppose we input a squeezed state with $\gamma=\operatorname{diag}\left(1 / r, \hbar^{2} r\right)$. Then the determinants of the covariance matrices of initial and final states will be $\hbar^{2}$ and $\hbar^{2}+\left(\hbar^{2} \Lambda \delta t / 2 r\right)$, respectively. The entropy change of the state can, thus, be made as small as desired by increasing the value of $r$, and so, the above bound can be saturated, leading to $W\left(\Omega_{\delta t}, H\right)=\Delta U$. Consequently, the maximum power at which a collapse engine could, in principle, operate is given by

$$
\frac{d W}{d t}=\frac{\hbar^{2} \Lambda}{4 m} .
$$

Using the upper range estimation $\Lambda \sim 10^{6} \mathrm{~s}^{-1} \mathrm{~m}^{-2}$, we have that a collapse engine powered by a single electron would produce $(d W / d t) \sim 10^{-32} \mathrm{~W}$. Assuming total control over the electrons of a macroscopic sample, one would need a kiloton of Hydrogen to power a 40W light bulb. 
Conclusion.-In this Letter, we addressed the problem of determining how much work can be extracted from operational-as opposed to state-resources. We proved that the solution to this problem in the asymptotic limit is given by a single-letter formula that quantifies the amount of distillable work that a channel can, in principle, generate when supplemented with thermal operations and catalyst states. Moreover, we found how this quantity can be determined for bosonic channels and computed it exactly for the case of the GRW dynamics, hence, determining the maximum power which a hypothetical collapse engine could provide for free.

Note that we have only studied operational resources regarding their capacity to generate work. An interesting topic for future research is to extend our results and draw a map of the interconversion relations between different operational resources. In the case of state resources, there is a unique monotonic quantity, the free energy, determining the optimal rates for state transformations [7]. In this Letter, we have identified an operational monotone, the distillable work, but we suspect that there may be many others.

We thank Ralph Silva and Noah Linden for useful discussions. M. N. acknowledges support from the Spanish MINECO Project No. FIS2013-40627-P.

[1] M. Horodecki, P. Horodecki, and J. Oppenheim, Phys. Rev. A 67, 062104 (2003).

[2] G. Gour and R. W. Spekkens, New J. Phys. 10, 033023 (2008).

[3] R. Horodecki, P. Horodecki, M. Horodecki, and K. Horodecki, Rev. Mod. Phys. 81, 865 (2009).

[4] G. Gour, M. P. Müller, V. Narasimhachar, R. W. Spekkens, and N. Yunger Halpern, arXiv:1309.6586.

[5] M. A. Nielsen and I. L. Chuang, Quantum computation and quantum information (Cambridge University Press, Cambridge, England, 2010).
[6] M. Horodecki and J. Oppenheim, Nat. Commun. 4, 2059 (2013)

[7] F. G. S. L. Brandão, M. Horodecki, J. Oppenheim, J. M. Renes, and R. W. Spekkens, Phys. Rev. Lett. 111, 250404 (2013).

[8] K. Takara, H.-H. Hasegawa, and D. Driebe, Phys. Lett. A 375, 88 (2010).

[9] M. Esposito and C. Van den Broeck, Europhys. Lett. 95, 40004 (2011).

[10] J. Åberg, Phys. Rev. Lett. 113, 150402 (2014).

[11] F. G. S. L. Brandao, M. Horodecki, N. H. Y. Ng, J. Oppenheim, and S. Wehner, Proc. Natl. Acad. Sci. U.S.A. 112, 3275 (2015).

[12] P. Skrzypczyk, A. J. Short, and S. Popescu, Nat. Commun. 5, 4185 (2014).

[13] See Supplemental Material at http://link.aps.org/ supplemental/10.1103/PhysRevLett.115.010405 for the proof that the distillable work can be superactivated, a characterization of gaussian channels with a finite amount of distillable work, and a proof of Proposition 2.

[14] Crucially, at the end of the regeneration step, the free energy of the reconstructed catalysts also tends to its initial value.

[15] V. Vedral, Rev. Mod. Phys. 74, 197 (2002).

[16] S. L. Braunstein and P. van Loock, Rev. Mod. Phys. 77, 513 (2005).

[17] M. M. Wolf, G. Giedke, and J. I. Cirac, Phys. Rev. Lett. 96, 080502 (2006).

[18] G. C. Ghirardi, A. Rimini, and T. Weber, Phys. Rev. D 34, 470 (1986).

[19] R. Penrose, Gen. Relativ. Gravit. 28, 581 (1996).

[20] L. Diósi, J. Phys. A 40, 2989 (2007).

[21] S. L. Adler, J. Phys. A 40, 2935 (2007).

[22] If we model the bath as a grey body, then it radiates energy at a rate of $\sigma\left(T^{4}-T_{c}^{4}\right)$, where $T_{c}$ is the temperature of the $\mathrm{CMB}$ and $\sigma$ is a constant that depends on how well isolated the bath is. The power transferred to the bath by the GRW dynamics is, on the other hand, independent of $T$ and proportional to $\Lambda$. It follows that the bath will reach a stationary temperature $T_{\text {eq }}$ whose exact value will depend on both $\sigma$ and $\Lambda$. 